\title{
Correction to: New and updated life cycle inventories for surfactants used in European detergents: summary of the ERASM surfactant life cycle and ecofootprinting project
}

\author{
Diederik Schowanek ${ }^{1}$ - Tilly Borsboom-Patel ${ }^{2}$ - Alain Bouvy ${ }^{3}$. John Colling ${ }^{4}$ Juan Antonio de Ferrer ${ }^{5}$. \\ Diana Eggers ${ }^{6} \cdot$ Karsten Groenke $^{7} \cdot$ Thomas Gruenenwald $^{8} \cdot$ Johanna Martinsson $^{9} \cdot$ Philip Mckeown $^{10}$. \\ Bjoern Miller ${ }^{11} \cdot$ Simon Moons ${ }^{12} \cdot$ Kate Niedermann $^{13} \cdot$ Miguel Pérez $^{14} \cdot$ Christine Schneider $^{15} \cdot$ Jean-Francois Viot $^{16}$
}

Published online: 5 February 2018

(C) Springer-Verlag GmbH Germany, part of Springer Nature 2018

\section{Correction to: Int J Life Cycle Assess}

https://doi.org/10.1007/s11367-017-1384-x

In Table 4, reference to the footnotes should be a, b and c (instead of 1,2 and 3).

Table 6 was not correctly displayed. The reference approach used in this study should be marked in bold (not in grey).

The online version of the original article can be found at https://doi.org/ 10.1007/s11367-017-1384-x

Diederik Schowanek

schowanek.d@pg.com

1 Procter \& Gamble, Brussels Innvovation Centre, Temselaan 100, 1853, Strombeek-Bever, Belgium

2 Shell Chemicals Europe BV, Weena 70, 3012 CM Rotterdam, The Netherlands

3 CEFIC, The European Chemical Industry Council, Avenue E. Van Nieuwenhuyse 4, 1160 Brussels, Belgium

4 Stepan Europe SAS, Chemin Jongkind, CS 20127, 38341 Voreppe Cedex, France

5 CEPSA QUIMICA S.A., Fabrica de Puente Mayorga, Apdo. Correos 40, 11360 San Roque, Cádiz, Spain

6 PE International AG (now thinkstep AG), Hauptstraße 111-113, 70771 Leinfelden-Echterdingen, Germany

7 Evonik Industries AG, Bäkerpfad 25, 47805 Krefeld, Germany

8 BASF, SE, Carl-Bosch-Str. 38, 67056 Ludwigshafen, Germany
Akzo Nobel Surface Chemistry, AB Stenunge Allé 3, SE-444 85 Stenungsund, Sweden

10 Unilever Safety \& Environmental Assurance Centre, Colworth Science Park Sharnbrook, Bedford MK44 1LQ, UK

11 Sasol Germany GmbH, Anckelmannsplatz 1, 20537 Hamburg, Germany

12 Huntsman Performance Products, Everslaan 45, 3078 Everberg, Belgium

13 Dow Chemical Company, Zurich, Switzerland

14 Kao Chemicals Europe, Puig dels Tudons no. 10, 08210 Barberà del Vallès, Barcelona, Spain

15 Henkel AG \& Co. KgaA, Henkelstrasse 67, 40589 Duesseldorf, Germany

16 Solvay Research \& Innovation, Centre Paris, 52 rue de la Haie Coq, 93308 Aubervilliers Cedex, France 


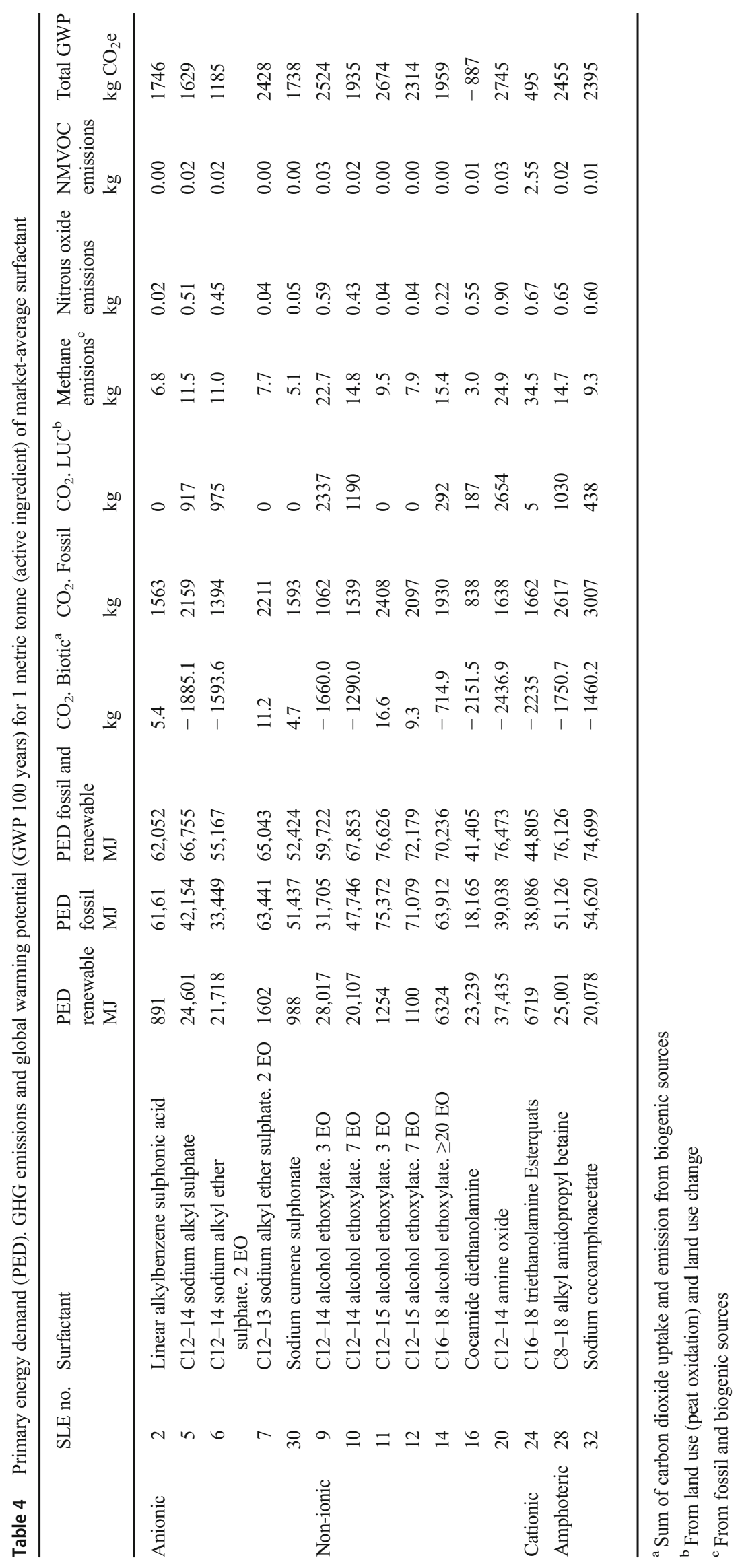


Table 6 Sensitivity related to allocation by mass vs. price

\begin{tabular}{|c|c|c|c|c|c|c|c|}
\hline & \multirow[t]{3}{*}{ Substance } & \multicolumn{3}{|c|}{ Primary energy demand } & \multicolumn{3}{|c|}{ Global warming potential } \\
\hline & & \multicolumn{3}{|c|}{$\mathrm{MJ} /$ tonne } & \multicolumn{3}{|c|}{ Tonne- $\mathrm{CO}_{2}$-equiv. / tonne } \\
\hline & & Price & Mass & Difference $(\%)$ & Price & Mass & Difference $(\%)$ \\
\hline 15 & Diethanolamine & 52,769 & 65,562 & +24 & 2220 & 2760 & +24 \\
\hline 17 & Dimethylamine & 69,874 & 79,220 & -12 & 2695 & 3066 & -12 \\
\hline 21 & Beef tallow & 24,782 & 274,613 & +1008 & -1529 & 28,253 & +1748 \\
\hline 22 & Triethanolamine & 72,086 & 71,437 & -1 & 3033 & 2669 & -12 \\
\hline 25 & Coconut oil methyl ester & 62,800 & 38,055 & +65 & 1193 & -1989 & +60 \\
\hline $25 \mathrm{a}$ & Palm oil methyl ester & 60,633 & 53,187 & +14 & 3030 & 2658 & +14 \\
\hline $25 b$ & Palm kernel oil methyl ester & 80,240 & 54,585 & +47 & 4017 & 2696 & +49 \\
\hline 27 & Chloroacetic acid & 33,905 & 29,794 & +14 & 1649 & 1450 & +14 \\
\hline 31 & Aminoethylethanolamine & 91,767 & 66,778 & +37 & 4095 & 2974 & +38 \\
\hline 32 & Sodium cocoamphoacetate & 81,160 & 74,699 & +9 & 2673 & 2395 & +12 \\
\hline
\end{tabular}

The reference approach used in this study is marked in bold. 